\title{
CONGENITAL ADRENAL HYPERPLASIA IN PATIENTS WITH DISORDERS OF SEXUAL DIFFERENTIATION
}

\author{
Warda Fatima* \\ Department of Microbiology \& Molecular Genetics, University of the Punjab, Quaid-e-Azam campus, Lahore.
}

Tayyaba Rafiq

Department of Microbiology \& Molecular Genetics, University of the Punjab, Quaid-e-Azam campus, Lahore.

Saqib Mahmood

Department of Allied health sciences, University of Health Sciences, Lahore.

*Corresponding author

Department of Microbiology \& Molecular Genetics, University of the Punjab, Quaid-e-Azam campus, Lahore.

Telephone: 03334386473; Email: warda.mmg@pu.edu.pk

\begin{abstract}
Congenital Adrenal Hyperplasia (CAH) is considered to be the most common cause of genital ambiguity in children. According to World's literature, 90-95\% of this disease is caused by 21-hydroxylase deficiency that impairs the synthesis of cortisol and aldosterone. The consequent excess in androgen production leads to virilization in the affected females. This study was aimed to find the number of cases with CAH (21hydroxylase deficiency) in the children presented with disorders of sexual differentiation. For this purpose, 100 patients presented to The Children's hospital for gender assessment were taken and their 17- $\mathrm{OH}$ progesterone levels were measured to confirm 21hydroxylase deficiency, and chromosomal analysis was done to confirm chromosomal sex. Results indicated that out of 100 patients 49 were suffering from CAH. $63.2 \%$ of $\mathrm{CAH}$ patients were initially presented as males. Out of these, $44.8 \%$ were reassigned female gender on chromosomal analysis. So, it is concluded that the majority of patients presented with genital ambiguity in the tertiary care health facility have the ambiguity due to congenital adrenal hyperplasia.
\end{abstract}

Key Words: Congenital adrenal hyperplasia, virilization, 17 hydroxy progesterone, chromosomal sex, Karyotype.

\section{Introduction}

Infants with a genital appearance that does not permit gender declaration at birth are said to have ambiguous genitalia or disorders of sexual differentiation. These disorders usually result in the conflict between chromosomal, gonadal, and phenotypic sex (Lee and Houk, 2008). Among many causes of genital ambiguity, Congenital adrenal hyperplasia is considered one of the most common (Bhanji et al., 2004). It is an autosomal recessive endocrine disorder (Khan et al., 2011; Hedi et al., 2005) in which there is a deficiency of one of the enzymatic activities necessary for cortisol synthesis from cholesterol in the adrenal cortex. Decreased levels of circulating cortisol result in increased secretion of adrenocorticotropic hormone 
(ACTH) from the anterior pituitary and unrestrained ACTH stimulation causes the hyperplasia of the adrenal cortex resulting in overproduction of the adrenal steroids that do not require the deficient enzyme activity, and deficiency of the steroids distal to the disrupted enzymatic step (Ambroziak et al., 2005). Overproduction of adrenal androgens in some of these disorders often results in prenatal virilization in females, with an ambiguous or male-like external genital tract at birth and precocious puberty that may occur in both sexes. In $95 \%$ of the cases of $\mathrm{CAH}$, there is a deficiency of the 21-hydroxylase (21-OH) enzyme. In 5-8\% of the cases, the deficiency is of 11-ß-hydroxylase while other defects constitute less than $1 \%$ of all reported cases (Bhanji et al., 2004).

The diagnosis of Congenital Adrenal Hyperplasia (CAH) due to 21-OH Deficiency is confirmed by biochemical findings, such as an unequivocally elevated serum concentration of 17-hydroxy progesterone (17-OHP). Ultrasound examination, serum 17-OHP levels, and chromosome analysis are necessary for true gender identity. This study was done to identify the frequency of CAH in patients with DSD presented to the children's hospital Lahore and also to categorize them as genotypic males or females by chromosome analysis.

\section{Subjects and Methods}

Patients referred to the Cytogenetics laboratory of the Children Hospital \& The Institute of Child Health Lahore, from the Departments of Pediatric Endocrinology, Urology and Surgery for gender identification through chromosomal analysis from January 2007 to January 2008 were included in the study. History of nausea, vomiting, weakness, darkening or pigmentation of the skin, acne, voice deepening, appearance of pubic hair, excessive hair growth, menstrual periods, and hirsutism was taken and complete genital examination was done. A pelvic ultrasound scan of each patient was carried out to determine the nature and condition of internal reproductive organs. After taking consent from the patients or parents of patients (in case of minor subjects) blood in heparin vacutainers was collected and chromosomal analysis with Giemsa staining method was done (Rooney and Czepulkowski, 1992). Blood samples from the patients were also taken for the serum 17-OH progesterone levels to confirm the diagnosis of congenital adrenal hyperplasia.

\section{Results}

A total of 100 patients with disorders of sexual differentiation were included in the study with ages ranged from 1day to 22 years with a mean age of $3.2 \pm 4.3$ years. Family history of ambiguous genitalia was present in 21/100 (21\%) patients, while 50 out of the total 100 patients (50\%) were found to be the product of consanguineous marriage. Gender assignment at the time of birth and after chromosome analysis is shown in Table 1. For the diagnosis of congenital adrenal hyperplasia, the serum 17-OH progesterone levels were measured and these were found elevated in 49 out of 100 (49\%) patients, thus they were diagnosed as having congenital adrenal hyperplasia ( 21 hydroxylase deficiency).General features of the CAH patients were also observed and it was noted that approximately $27(55 \%) \mathrm{CAH}$ patients also had complaints of vomiting. Respiratory distress was observed in 19 (38.7\%), 16 (32.6\%) were lethargic, 
Table 1: Relation between genotype and phenotype of patients with ambiguous genitalia.

\begin{tabular}{cccc}
\hline $\begin{array}{c}\text { Phenotype assigned at } \\
\text { birth }\end{array}$ & $\begin{array}{c}\text { No of Cases } \\
(\mathbf{n = 1 0 0})\end{array}$ & Chromosomal sex & $\begin{array}{c}\text { Phenotype after } \\
\text { chromosome analysis }\end{array}$ \\
\hline Male & 39 & $46, \mathrm{XY}$ & Male \\
\hline Male & 25 & $46, \mathrm{XX}$ & Female \\
\hline Female & 11 & $46, \mathrm{XY}$ & Male \\
\hline Female & 18 & $46, \mathrm{XX}$ & Female \\
\hline None & 03 & $46, \mathrm{XX}$ & Female \\
\hline None & 01 & $46, \mathrm{XY}$ & Male \\
\hline Male & 01 & $46, \mathrm{XXY}$ & Male \\
\hline Male & 01 & $46, \mathrm{X}$ del $(\mathrm{Xq})$ & Female \\
\hline
\end{tabular}

and $9(18.3 \%)$ showed abnormal pigmentation of the body. All of these parameters observed are listed in Table 2. The gender assignment of 49 patients, diagnosed as having $\mathrm{CAH}$, before and after chromosome analysis is shown in Table 3 .

Table 2: General features of patients with CAH (Congenital Adrenal Hyperplasia).

\begin{tabular}{lc}
\hline Features & No of patients with positive results \\
\hline Frequent Vomiting & $27(55.1 \%)$ \\
\hline Respiratory distress & $19(38.7 \%)$ \\
\hline Fits & $06(12.2 \%)$ \\
\hline Lethargy & $16(32.6 \%)$ \\
\hline Greasy hair \& Skin & $04(8.1 \%)$ \\
\hline Increased pigmentation & $09(18.3 \%)$ \\
\hline Failure to thrive & $10(20.4 \%)$ \\
\hline Hirsutism & $05(10.2 \%)$ \\
\hline
\end{tabular}

Table 3: Relation between genotype and phenotype of patients with CAH.

\begin{tabular}{cccc}
\hline $\begin{array}{c}\text { Phenotype assigned at } \\
\text { birth }\end{array}$ & CAH $(\mathbf{n}=49)$ & Chromosomal sex & $\begin{array}{c}\text { Phenotype after } \\
\text { chromosome analysis }\end{array}$ \\
\hline Male & 09 & $46, \mathrm{XY}$ & Male \\
\hline Male & 22 & $46, \mathrm{XX}$ & Female \\
\hline Female & 01 & $46, \mathrm{XY}$ & Male \\
\hline Female & 12 & $46, \mathrm{XX}$ & Female \\
\hline None & 03 & $46, \mathrm{XX}$ & Female \\
\hline None & 01 & $46, \mathrm{XY}$ & Male \\
\hline
\end{tabular}

\section{Discussion}

As the main objective of this study was to find out the frequency of CAH in patients presenting with genital ambiguity and also to find out the male and female ratio of this disease, so 100 patients with ambiguous genitalia, who were referred to the cytogenetics laboratory of The Children's Hospital for correct gender assessment, were examined. Consanguinity in parents was seen in 55\% of patients with 
$\mathrm{CAH}$. This conforms to the fact that congenital adrenal hyperplasia is inherited in an autosomal recessive manner and consanguinity increases the chances of having a child with this defect (Bonfig et al., 2007; Hedi et al., 2005). In a study by Joshi et al., (2006) only 25\% of the patients were the product of consanguineous marriages, this may be due to the low level of consanguineous marriages in that population (Joshi, Rao and Desai, 2006). Family history of the same disease was also noted in $22.4 \%$ of the CAH patients that is consistent with the study of Sido et al. (2005) from the Romanian population that has a family history of $\mathrm{CAH}$ in 10 out of 38 patients presented (26.3\%). In the present study, $65.3 \%$ of the diagnosed CAH patients were initially assigned male gender at the time of birth this conforms with a study from India which reported male gender assignment at birth to $60 \%$ of patients in Indian literature (Joshi, Rao and Desai, 2006) but unlike that reported from other populations, $46.5 \%$ patients presented as males while $53.5 \%$ as females in the population of Jordan (Al-Maghribi, 2007). This may be due to social preference in South Asian societies to a male gender over females resulting in females with even slight ambiguity assigned a male gender. Because serum 17-hydroxyprogesterone (17OHP) is distinctly elevated in 21OHD, it stands as the classical steroid marker for this disease (Tonetto-Fernandes et al., 2006). In our study, congenital adrenal hyperplasia was diagnosed by elevated levels of serum 17-OH progesterone. It was observed in this study that CAH was the single most common cause (49\%) of ambiguous genitalia. $\mathrm{CAH}$ being the commonest etiological factor causing genital ambiguity has been reported in other populations also (Joshi, Rao and Desai, 2006; Nimkam, 2002). Some studies reported as high as $72 \%$ of patients with CAH in their cases (Al Agha, Thomsett and Batch, 2001). High rates of failure to thrive, recurrent vomiting, hyperpigmentation, and hirsutism, as were found in our case. This favours the diagnosis of 21-hydroxylase deficiency and warrants steroid replacement after measuring levels of 17 OHP (Bajpai, Kabra and Menon, 2004). The extent of ambiguity in girls in this study was not influenced by the form of disease or by the age at diagnosis. This suggests that prenatal androgen exposure is the primary determinant of the extent of ambiguity. Postnatal androgen exposure can cause mild clitoral enlargement and virilization (Bajpai, Kabra and Menon, 2004). Most of the results of our study are consistent with scientific literature from other populations confirming that $\mathrm{CAH}$ (21 hydroxylase deficiency) is the major cause of genital ambiguity in children. In the present study, we only diagnosed CAH patients due to deficiency of 21Hydroxylase enzyme. Further studies are required to diagnose CAH patients due to deficiencies of other enzymes. Moreover, there is a need to establish DNA based diagnosis to identify the carriers of this disease and to offer a prenatal diagnosis to the parents in their next pregnancies. A thorough diagnostic workup protocol should be established in our population to make an early and accurate diagnosis in the affected children so that they are assigned the correct gender and plans could be made for their long-term management.

\section{Conclusion}

It can be concluded from these results that congenital adrenal hyperplasia is the single most common cause of genital ambiguity in patients presenting with ambiguous genitalia, and it is the 
virilization of the female external genitalia that is the cause of initial ambiguity, in the majority of the cases.

\section{References}

Al Agha, A.E., Thomsett, M.J., Batch, J.A., 2001. The children of uncertain sex: 17 years of experience. Journal of Pediatrics and Child Health, 37: 348-351.

Al-Maghribi, H., 2007. Congenital Adrenal Hyperplasia: Problems with Developmental Anomalies of the External Genitalia and Sex Assignment. Saudi Journal of Kidney Diseases and Transplantation, 18(3): 405-413.

Ambroziak, U., Bednarczuk, T., Ginalska-Malinowska, M., Małunowicz, E.M., Grzechocińska, B., Kamiński, P., Bablok, L., Przedlacki, J., Bar-Andziak, E., 2005. Congenital adrenal hyperplasia due to 21-hydroxylase deficiency - management in adults. Polish Journal of Endocrinology, 61 (1): $142-155$.

Bajpai, A., Kabra, M., Menon, P.S.N., 2004. 21-Hydroxylase Deficiency: Clinical Features, Laboratory Profile and Pointers to Diagnosis in Indian Children. Indian Pediatrics, 41: 1226-1232.

Bhanji, R., Khan, A.H., Balouch, I.L., Sabir, S., Nazir, Z., Billoo, A.G., 2004. Profile of Children with Congenital Adrenal Hyperplasia - a Hospital Study. Pakistan Journal of Medical Association, 54(10): 509-512.

Bonfig, W., Bechtold, S., Schmidt, H., Knorr, D., Schwarz, H.P., 2007. Reduced Final Height Outcome in Congenital Adrenal Hyperplasia under Prednisone Treatment: Deceleration of Growth Velocity during Puberty. The Journal of Clinical Endocrinology and Metabolism, 92(5): 1635-1639.

Hedi, L., Grinten, C.D., Noordam, K., Borm, G.F., Otten, B.J., 2005. Absence of Increased Height Velocity in the First Year of Life in Untreated Children with Simple Virilizing Congenital Adrenal Hyperplasia. The Journal of Clinical Endocrinology and Metabolism, 91(4): 1205-1209.

Joshi, R.R., Rao, S., Desai, M., 2006. Etiology and Clinical Profile of Ambiguous genitalia - An Overview of 10 Years Experience. Indian Pediatrics, 43: 974-979.

Khan, A.H., Aban, M., Raza, J., Haq, N., Jabbar, A., Moatter, T., 2011. Ethnic disparity in 21 -hydroxylase gene mutations identified in Pakistani congenital adrenal hyperplasia patients. BMC Endocrine Disorders, 11: 5 .

Lee, P.A., Houk, C.P., 2008. Disorders of Sexual Differentiation in the Adolescent. Annals of the New York Academy of Sciences, 1135: 67-75.

Nimkam, S., Likitmoskul, S., Sangecharoenkit, P., Pathomvanich, A., Sawathiparnich, P., Wacharasindhu, S., 2002. Ambiguous genitalia: An overview of 22 years experience and the diagnostic approach in the Pediatric department, Siriraj Hospital. Journal of the Medical Association of Thailand, 85: 496-505.

Rooney, D.E. and Czepulkowski, B.H., 1992. Human Cytogenetics constitutional analysis a physical approach Vol I. Oxford: Oxford University Press, pp. 95-96. 
Sido, A.G., Weber, M.M., Sido, P.G., Clausmeyer, S., Heinrich, U., Schulze, E., 2005. 21 - Hydroxylase and 11_-Hydroxylase Mutations in Romanian Patients with Classic Congenital Adrenal Hyperplasia. The Journal of Clinical Endocrinology and Metabolism, 90(10): 5769-5773.

Tonetto-Fernandes, V., Lemos-Marini, S.H.V., Kuperman, H., Ribeiro-Neto, L.M., Verreschi, I.T.N., Kater, C.E., the Brazilian Congenital Adrenal Hyperplasia Multicenter Study Group., 2006. Serum 21-Deoxycortisol, 17-Hydroxyprogesterone, and 11-Deoxycortisol in Classic Congenital Adrenal Hyperplasia: Clinical and Hormonal Correlations and Identification of Patients with 11_Hydroxylase Deficiency among a Large Group with Alleged 21- Hydroxylase Deficiency. The Journal of Clinical Endocrinology and Metabolism, 91(6): 2179-2184. 